\title{
A Wind-Tunnel Investigation of Wind-Turbine Wakes: Boundary-Layer Turbulence Effects
}

\author{
Leonardo P. Chamorro • Fernando Porté-Agel
}

Received: 22 October 2008 / Accepted: 30 March 2009 / Published online: 23 April 2009

(C) The Author(s) 2009. This article is published with open access at Springerlink.com

\begin{abstract}
Wind-tunnel experiments were performed to study turbulence in the wake of a model wind turbine placed in a boundary layer developed over rough and smooth surfaces. Hot-wire anemometry was used to characterize the cross-sectional distribution of mean velocity, turbulence intensity and kinematic shear stress at different locations downwind of the turbine for both surface roughness cases. Special emphasis was placed on the spatial distribution of the velocity deficit and the turbulence intensity, which are important factors affecting turbine power generation and fatigue loads in wind energy parks. Non-axisymmetric behaviour of the wake is observed over both roughness types in response to the non-uniform incoming boundary-layer flow and the effect of the surface. Nonetheless, the velocity deficit with respect to the incoming velocity profile is nearly axisymmetric, except near the ground in the far wake where the wake interacts with the surface. It is found that the wind turbine induces a large enhancement of turbulence levels (positive added turbulence intensity) in the upper part of the wake. This is due to the effect of relatively large velocity fluctuations associated with helicoidal tip vortices near the wake edge, where the mean shear is strong. In the lower part of the wake, the mean shear and turbulence intensity are reduced with respect to the incoming flow. The non-axisymmetry of the turbulence intensity distribution of the wake is found to be stronger over the rough surface, where the incoming flow is less uniform at the turbine level. In the far wake the added turbulent intensity, its positive and negative contributions and its local maximum decay as a power law of downwind distance (with an exponent ranging from -0.3 to -0.5 for the rough surface, and with a wider variation for the smooth surface). Nevertheless, the effect of the turbine on the velocity defect and added turbulence intensity is not negligible even in the very far wake, at a distance of fifteen times the rotor diameter.
\end{abstract}

Keywords Roughness effects · Turbulence - Wake structure - Wind-tunnel experiment · Wind turbine

L. P. Chamorro · F. Porté-Agel ( $\varangle)$

Saint Anthony Falls Laboratory, Department of Civil Engineering, University of Minnesota, 23rd Ave. SE, Minneapolis, MN 55414, USA

e-mail: fporte@umn.edu 


\section{Introduction}

Wakes of horizontal-axis wind turbines are complex turbulent flow structures with rotational motion induced by the turbine blades, longitudinal and radial pressure gradients, and spiral vortices originating from tip vortices shed by the blades. From a wind engineering perspective, there are two characteristics of wind turbine wakes that are of considerable practical interest: (a) the velocity deficit, which is related to the power loss from the wind turbine; and (b) the turbulence levels, which may affect flow-induced rotor loads on other turbines located downwind in a wind park. Understanding these effects is among the main reasons why turbine wakes have been the subject of intensive research, both experimentally and numerically, during the last few decades. A comprehensive review on wind-turbine-wake research is given by Vermeer et al. (2003).

Detailed understanding of the turbulent properties of turbine wakes under different inflow and surface conditions is important to maximize the energy production and ensure the structural integrity of the wind turbines. In fact, the ability to predict the spatial distribution of the mean velocity deficit is essential to optimizing the design (turbine siting) of wind farms. Understanding the spatial distribution of turbulence intensity generated inside turbine wakes is also important in determining fatigue loads on other wind turbines located downwind. In addition, a complete characterization of the turbulent properties in turbine wakes is desirable for validating and guiding the development of subgrid-scale parameterizations in numerical models of turbine wakes (e.g., large-eddy simulation (LES)).

The structure of wind turbines is known to be affected by the characteristics of the incoming flow. For example, Sheinman and Rosen (1992) have shown that neglecting the effect of turbulence in the incoming flow can lead to an overestimation of turbine output by more than 10\%. A wind-tunnel study performed by Medici and Alfredsson (2006) has shown that the wake of a model turbine located in the free stream is substantially changed by the presence of turbulence in the flow. It is important to note that one expects even stronger differences in the structure and dynamics of the wake if the turbine is located inside (totally or partially) of the surface layer of a turbulent boundary layer. This layer is characterized by strong vertical gradients of velocity and turbulence intensity. The spatial variability in the incoming flow (not present in free stream flows), together with the anisotropy imposed by the presence of the ground, are expected to produce a non-axisymmetric wake structure. Among the factors that affect the incoming flow characteristics and, therefore, the wake structure are the aerodynamic surface roughness, the presence of surface inhomogeneities (e.g., surface roughness and temperature transitions), topography, and the effects of the wakes from other turbines located upwind in a wind park.

Turbulence intensity $\left(I_{u}\right)$ plays a direct role on the mean force $(\bar{F})$ and bending moment $(\bar{M})$ acting on the turbine (Binh et al. 2008), and is commonly defined as the standard deviation of the wind velocity component in the average wind direction $\left(\sigma_{u}\right)$ divided by the wind velocity at the turbine hub height $\left(U_{h u b}\right)$ :

$$
I_{u}=\frac{\sigma_{u}}{U_{h u b}} .
$$

In order to understand the effect of the turbulence intensity on the mean force $\bar{F}$, let us consider the instantaneous force acting on an element $d A$ of the turbine:

$$
d F=\frac{1}{2} \rho C_{f} d A(U+u)^{2}=\frac{1}{2} \rho C_{f} d A\left(U^{2}+2 U u+u^{2}\right),
$$


where $\rho$ is the air density, $C_{f}$ is the aerodynamic force coefficient, $d A$ is the cross-sectional area of the element, $U$ is the mean wind velocity magnitude, and $u$ is the turbulent velocity fluctuation. After time averaging, the mean force becomes:

$$
\overline{d F}=\frac{1}{2} \rho C_{f} d A\left(U^{2}+\sigma_{u}^{2}\right),
$$

and if the flow is uniform, $U$ and $I_{u}$ are constant and the total force over the turbine is:

$$
\bar{F}=\frac{1}{2} \rho\left\langle C_{f}\right\rangle A U^{2}\left(1+I_{u}^{2}\right),
$$

where \langle\rangle represents a spatial average over the turbine cross-sectional area.

Following a similar approach, the bending moment $\bar{M}$ can be expressed as:

$$
\bar{M}=\int_{0}^{H} \frac{1}{2} \rho C_{f} U^{2}\left(1+I_{u}^{2}\right) c(\xi) \xi d \xi
$$

where $c(\xi)$ is the characteristic size of the turbine element at position $\xi$ and $H$ denotes the wind turbine height. Note that Eq. 4 assumes a uniform distribution of $U$ and $I_{u}$ in the incoming flow. This is the case of free-stream flows. However, in the case of a wind turbine in a boundary-layer flow, characterized by non-uniform distributions of $U$ and $I_{u}$ in the surface layer, using that equation with a single value of $U$ and $I_{u}$ could lead to important errors.

As pointed out by Rosen and Sheinman (1996), turbulence intensity is commonly used as a measure of the fatigue accumulation on various components of the turbine. In general, models for turbulence levels in turbine wakes are limited to single-point values or averaged magnitudes, thus not taking into account any cross-sectional variability. In particular, it is common practice to consider turbulence intensity at the hub of the wind turbine as representative for the whole rotor.

Several models have been proposed to estimate the so-called added turbulence intensity, $I_{+}$, which is defined as a function of the ambient turbulent intensity, $I_{0}$, and the turbulence intensity in the wake $I_{\text {wake }}$. In particular,

$$
I_{\text {wake }}^{2}=I_{0}^{2}+I_{+}^{2} \Rightarrow I_{+}=\sqrt{I_{\text {wake }}^{2}-I_{0}^{2}} .
$$

Quarton and Ainslie (1989) suggested the following empirical expression for the added turbulence $\left(I_{+}\right)$produced by the action of the rotor in a free-stream flow:

$$
I_{+}=4.8 C_{T}^{0.7} I_{0}^{0.68}\left(x / x_{n}\right)^{-0.57}
$$

where $C_{T}$ is the turbine thrust coefficient and $x_{n}$ is the length of the near-wake region. This length is defined, according to Vermeulen (1980), as:

$$
x_{n}=\frac{\sqrt{0.214+0.144 m}(1-\sqrt{0.134+0.124 m})}{(1-\sqrt{0.214+0.144 m}) \sqrt{0.134+0.124 m}} \frac{r_{0}}{(d r / d x)}
$$

where $m=\frac{1}{\sqrt{1-C_{T}}}, r_{0}=R \sqrt{\frac{m+1}{2}}$ and $R$ is the rotor radius.

The wake growth rate, $d r / d x$, is given by the contributions of the ambient turbulence, $(d r / d x)_{a}$, the shear-generated turbulence, $(d r / d x)_{m}$, and the mechanical turbulence, $(d r / d x)_{\lambda}$, and defined as:

$$
d r / d x=\sqrt{\left(\frac{d r}{d x}\right)_{a}^{2}+\left(\frac{d r}{d x}\right)_{m}^{2}+\left(\frac{d r}{d x}\right)_{\lambda}^{2}},
$$


where each contribution is estimated as $\left(\frac{d r}{d x}\right)_{a}^{2}=2.5 I_{0}+0.005,\left(\frac{d r}{d x}\right)_{m}^{2}=\frac{(1-m) \sqrt{1.49+m}}{9.76(1+m)}$ and $\left(\frac{d r}{d x}\right)_{\lambda}^{2}=0.012 B \lambda . B$ is the number of blades and $\lambda$ is the tip speed ratio, which is defined as the ratio between the speed of the tip blade and the velocity of the incoming flow.

Crespo and Hernandez (1996), based on both experimental and numerical approaches, proposed the following relation for the parameter ranges $5<x / d<15,0.07<I_{u}<0.014$ and $0.1<a<0.4$, where $a$ is the induced velocity factor and $d$ is the rotor diameter:

$$
I_{+}=0.73 a^{0.8325} I_{0}^{0.0325}(x / d)^{-0.32} .
$$

Although they used the induced velocity factor $a$ instead of the thrust coefficient $C_{T}$ proposed by Quarton and Ainslie (1989), these two are related. Specifically, according to the actuator disk theory, for $a<0.5$, the relation between the induced velocity factor $a$ and the thrust coefficient is $C_{T}=4 a(1-a)$. Hassan (1992), based on further work, proposed a similar expression for the added turbulence:

$$
I_{+}=5.7 C_{T}^{0.7} I_{0}^{0.68}\left(x / x_{n}\right)^{-0.96},
$$

and under a wind-farm configuration, Frandsen and Thogersen (1999) proposed a model for the added turbulence that takes into account the wind-farm layout. The model is based on the geostrophic drag law and takes into account the additional 'surface roughness' generated by the turbines:

$$
I_{+}=\frac{1}{2}\left(I_{0}+\sqrt{I_{0}^{2}+I_{++}^{2}}\right)
$$

where

$$
I_{++}=\frac{0.36}{1+0.2 \sqrt{s_{1} s / C_{T}}} .
$$

Here $s_{1}$ and $s$ are the inter-turbine spacings (normalized by the rotor diameter) within a row and between rows, respectively. The Frandsen and Thogersen (1999) model applies above the hub height, and has become the European standard. However, as pointed out by Burton et al. (1995), to date there is no well-validated model for the prediction of turbulence intensity inside wind farms.

In this paper, the effect of boundary-layer flow on turbine wake characteristics is studied using detailed wind-tunnel measurements. Particular emphasis is placed on studying the distribution of streamwise mean wind velocity (and corresponding velocity deficit), turbulence intensity and kinematic shear stress in cross-sectional planes perpendicular to the axis of the wake at different downwind locations ( $x / d=3,5,10$ and 15, where $d$ is the turbine diameter). Experiments were carried out under neutral stability conditions over both rough and smooth surfaces in order to study the effect of roughness and the resulting velocity and turbulence fields on the wake characteristics. First, the wind-tunnel experiments are presented in Sect. 2. In Sect. 3, the spatial distribution of flow statistics measured in the near and far regions of the turbine wake are analyzed for both the rough and smooth cases. Finally, a summary is given in Sect. 4.

\section{Experimental Set-up}

A model 3-blade wind turbine was placed in the boundary-layer wind tunnel of the Saint Anthony Falls Laboratory at the University of Minnesota with the goal of studying turbulence 
characteristics of the turbine wake inside a fully developed neutrally-stratified boundary layer over both rough and smooth surfaces. The boundary-layer wind tunnel has a plan length of $37.5 \mathrm{~m}$ with a main test section fetch of $16 \mathrm{~m}$. There is a contraction with a $6.6: 1$ area ratio upwind of the test section along with flow conditioning/turbulence control consisting of a coarse wire mesh and honeycomb flow-straightened. The tunnel is driven by a 200 h.p. fan and is operated as a closed return loop. The turbulence intensity in the centre (free stream) of the wind tunnel is approximately $1 \%$ for a $2.5 \mathrm{~m} \mathrm{~s}^{-1}$ free-stream velocity. More details on the wind tunnel can be found in Carper and Porté-Agel (2008).

Mean wind velocity in the tunnel free stream was measured using Pitot static tubes, mainly for calibration purposes. Constant temperature hot-wire anemometry (CTA) was used to obtain high-resolution measurements of the streamwise and vertical components of the wind velocity. The probe is made of $5.0 \mu \mathrm{m}$ tungsten wires that are connected to an A.A. Lab Systems AN-1003 10-channel CTA system. During the calibration and measurements the temperature fluctuations were kept within a $\pm 0.2^{\circ} \mathrm{C}$ range to avoid bias errors due to thermal drift of the voltage signal.

As shown in Fig. 1, the rough surface was created by placing straight chains of approximately $5 \mathrm{~mm}$ height covering $10 \mathrm{~m}$ of the tunnel test section. The chains were aligned perpendicular to the flow direction and separated from each other by $0.20 \mathrm{~m}$. This roughness set-up is ideal to incorporate thermal effects through heating or cooling of the test section floor. A similar roughness configuration was recently used by Ohya (2001) to study stable boundary layers over rough surfaces. The smooth surface consists of a set of aluminum plates of $0.3 \mathrm{~m}$ length, each of which has a temperature control system to ensure the desired temperature level. Future experiments will use this heating/cooling system to study the effect of buoyancy (positive and negative) on the incoming boundary-layer wind and turbulence levels and, in turn, on the turbine wake characteristics.

A turbulent boundary layer was developed with the help of a tripping mechanism (40-mm picket fence) located at the exit of the wind-tunnel contraction, where the test section begins. The turbulent boundary layer was allowed to grow in zero pressure gradient conditions by adjustment of the wind-tunnel ceiling. The resulting boundary layer has a well-developed surface layer with constant shear stress and a logarithmic velocity profile.

The experiments were conducted at $2.5 \mathrm{~m} \mathrm{~s}^{-1}$ freestream flow velocity and a turbulent boundary-layer depth of $\delta \approx 0.4 \mathrm{~m}$ at the turbine location. It is important to note that these flow conditions are also reasonable for future experiments that will aim at extending the analysis presented here to include the effects of thermal stratification on the wake structure. The wind turbine consists of a three-blade GWS/EP-6030x3 rotor attached to a small DC generator. The turbine angular velocity can be adjusted by changing the resistance of the generator. During the experiments, the tip speed ratio was approximately $\lambda=4.4$ and $\lambda=4.2$ for the smooth and rough cases, respectively.

The tip speed ratio was adjusted to match that of field-scale turbines (usually between 3.5 and 6). As shown in Fig. 1, the bottom tip of the turbine was set to a height of 0.53 times the turbine radius, which is similar to that found in large-scale turbines $(\geq 2 \mathrm{MW})$. The turbine rotor was within the lowest half of the turbulent boundary layer. Despite the scaling issues associated with the difference in Reynolds number between the wind-tunnel flow and the atmospheric boundary-layer flow, our measurements provide detailed key information about the qualitative behaviour of turbine wakes in turbulent boundary-layer flows. It is also important to note that the high-resolution spatial and temporal measurements presented here can be used to systematically test the performance of numerical models (e.g., LES with different subgrid-scale models and wind turbine forces parameterizations) in the simulation of wind turbine wakes in turbulent boundary-layer flows. 

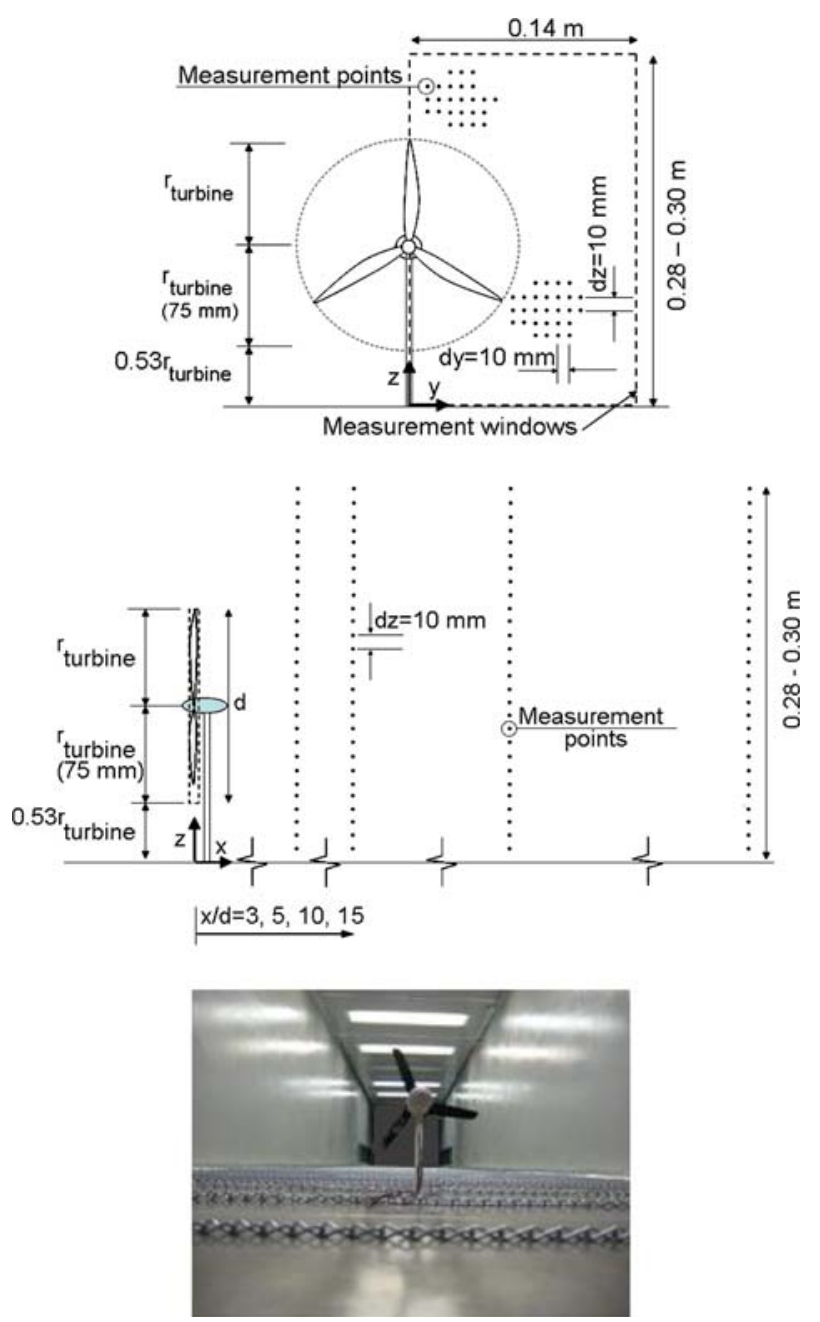

Fig. 1 Schematic of the wind turbine (front and side views), coordinate system and measurement locations (top and middle), and photograph of the test section with the wind turbine (bottom)

As shown in Fig. 1, the cross-wire anemometer was placed at different positions downwind of the turbine $(x / d=3,5,10$ and 15). At each location detailed measurements were collected in a plane perpendicular to the mean flow direction. Specifically, measurements of streamwise and vertical velocity components were taken in a plane that spans from $z=0$ to $0.28 \mathrm{~m}$ in the vertical direction, and from $y=0$ to $0.14 \mathrm{~m}$ in the spanwise direction. On that plane, measurements were taken over a grid of points separated by $d y=d z=10 \mathrm{~mm}$ (spanwise and vertical direction). More than 1400 measurement locations were considered to characterize the turbine wake for each surface roughness case. At each location, the sensor voltage signature was sampled at a rate of $1 \mathrm{kHz}$ for a measurement period ranging from 30 to $60 \mathrm{~s}$. An ensemble average of two experiments was performed to reduce aleatory uncertainties.

The zero pressure gradient boundary layer, developed upstream of the transition, had a Reynolds number based on the friction velocity: $R e_{v}=u_{*} \delta / v \approx 0.4 \times 10^{4}$, a friction velocity of $u_{* r}=0.16 \mathrm{~m} \mathrm{~s}^{-1}$ and $u_{* s}=0.11 \mathrm{~m} \mathrm{~s}^{-1}$ for the rough and smooth surface, 

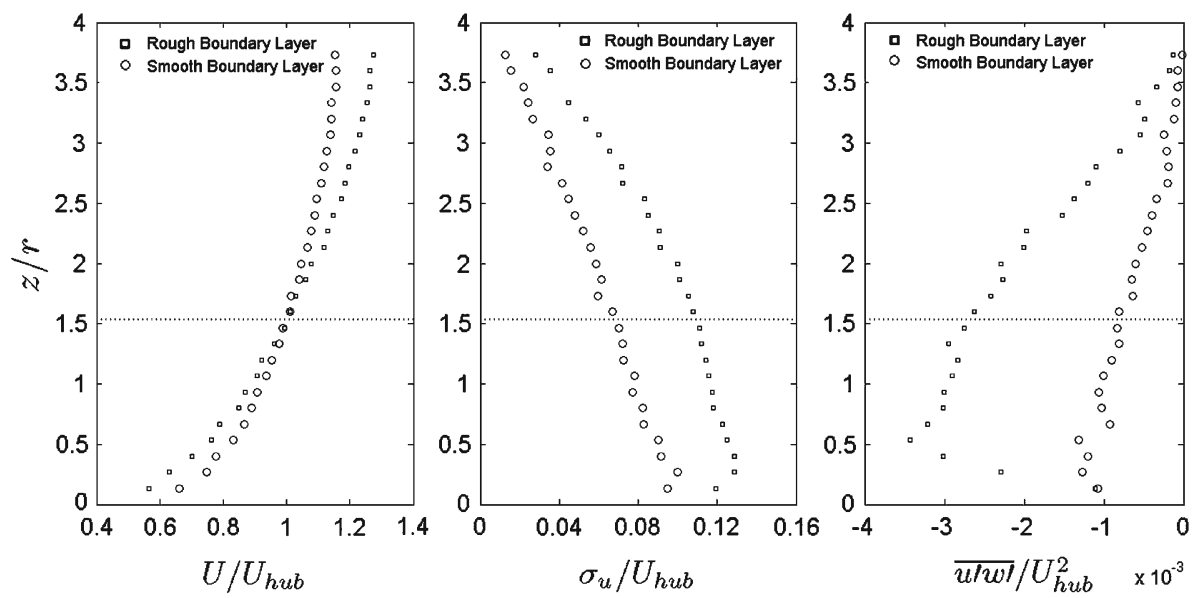

Fig. 2 Background characteristics of the turbulent boundary layer over both rough and smooth surfaces: normalized mean velocity (left), turbulence intensity (middle) and kinematic shear stress (right). The horizontal lines represent the turbine axis. Height is normalized with the turbine radius $r$

respectively. The aerodynamic surface roughness lengths were found to be $z_{0 r}=1.2 \mathrm{~mm}$ and $z_{0 s}=0.05 \mathrm{~mm}$. These parameters $\left(u_{*}\right.$ and $\left.z_{0}\right)$ were obtained by fitting a logarithmic velocity profile to the measured average velocity in the surface layer (approximately lowest $15 \%$ of the boundary layer). Figure 2 shows the vertical profiles of the mean velocity, turbulence intensity and turbulent stress measured in the boundary layer over the rough and smooth surfaces.

Calibration of the crosswire anemometer was performed at the beginning of each experimental run. Also a post-experiment calibration was carried out to check the validity of the calibration throughout the experiment. The anemometer was calibrated in the free-stream region against a Pitot-static probe, considering seven sensor inclination angles and seven wind velocities at each position. A cubic-spline table calibration method was then used to determine the two instantaneous velocity components from the two instantaneous voltage signatures. For more details on the calibration procedure, see Bruun (1995).

\section{Wake Characteristics}

\subsection{Wake in a Rough-Wall Boundary Layer}

A detailed cross-sectional characterization of the turbine wake over the rough surface is obtained from the high-resolution cross-wire anemometry measurements collected at different positions downwind of the turbine $(x / d=3,5,10$ and 15, see Fig. 1). Specifically, Figs. 3, 4 and 5 show the cross-sectional distribution $(y-z$ plane $)$ of the average streamwise velocity $(U)$, turbulence intensity $\left(I_{u}\right)$ and the kinematic shear stress $\left(\overline{u^{\prime} w^{\prime}}\right)$. Our results show nearly symmetric behaviour of mean velocity and turbulence intensity around $y=0$. For this reason, most of our measurements focused on one side $(y>0)$ of the wake. However, the kinematic shear stress showed roughly antisymmetric behaviour, with its axis of antisymmetry rotated approximately $45^{\circ}$. That behaviour is illustrated in Fig. 6, which shows an entire cross-sectional distribution of the kinematic stress at $x / d=5$.

Due to the effects of the non-uniform incoming boundary-layer velocity profile (logarithmic type) and the presence of the surface, the velocity distribution inside the turbine 

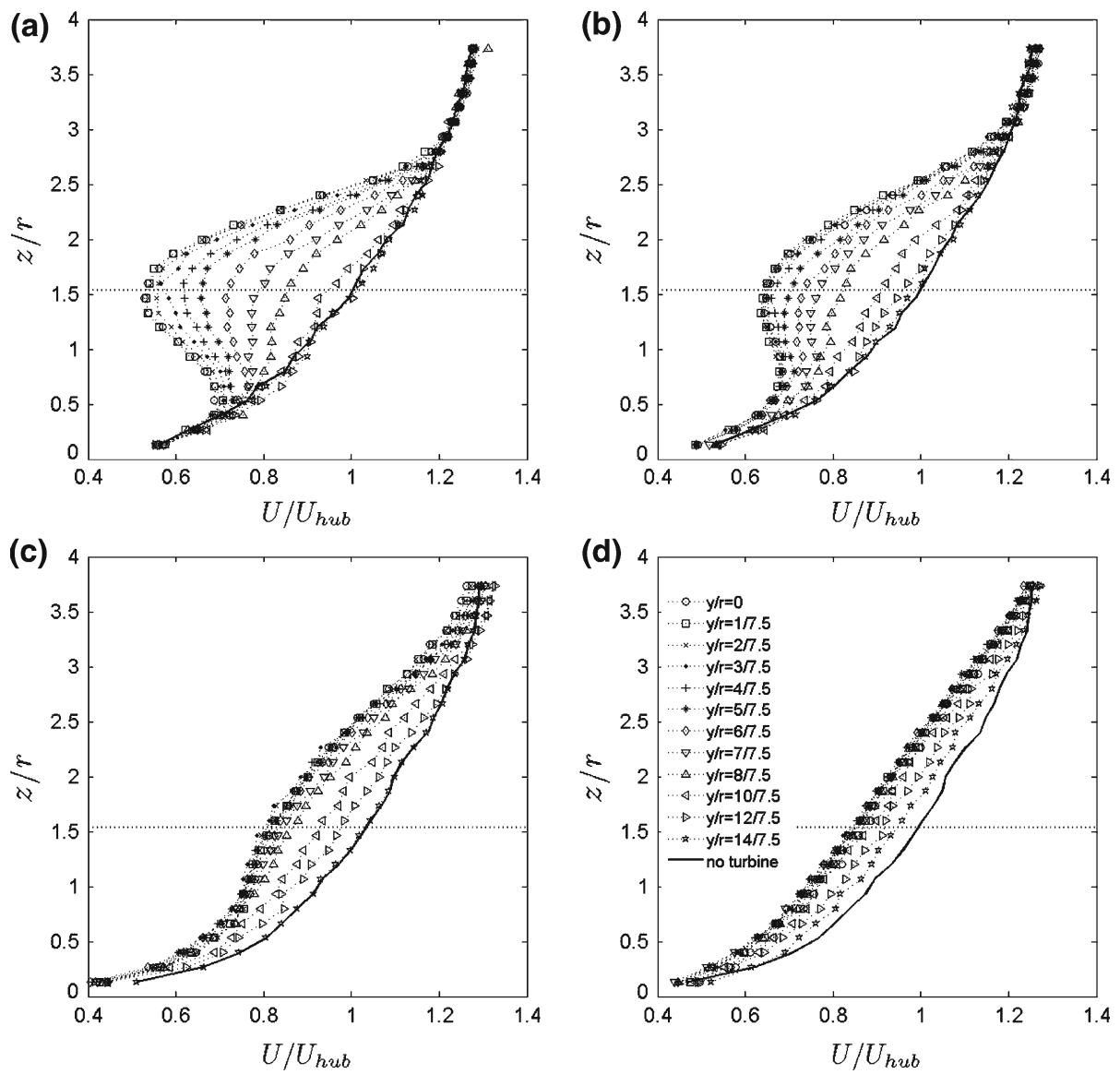

Fig. 3 Non-dimensional cross-sectional distribution of the mean velocity at different downwind positions inside the wake over the rough surface. $\mathbf{a} x / d=3 ; \mathbf{b} x / d=5 ; \mathbf{c} x / d=10$; and $\mathbf{d} x / d=15$. The horizontal line represents the turbine axis

wake (Fig. 3) does not show axisymmetric behaviour reported by previous studies in the case of free-stream flow (e.g., Crespo et al. 1999; Medici and Alfredsson 2006). The velocity reduction, modulated by the turbine, is superimposed on the logarithmic distribution of the incoming velocity, leading to a non-axisymmetric wake. However, as shown in Fig. 7, the velocity deficit calculated with respect to the incoming wind velocity distribution, $\Delta U_{x}=$ $U(x>0, y, z)-U(x<0, y, z)$, is approximately axisymmetric, with its axis of symmetry located slightly above the hub height. This is particularly relevant as it seems to point to the possibility of generalizing velocity-deficit formulations, developed for free-stream flow conditions, to non-uniform flows. As shown in Fig. 7, the axisymmetry breaks down near the surface downwind of the position at which the wake has grown enough to reach the surface. In this case, that distance is close to $x / d=5$.

The effect of the turbine is expected to lead to an enhancement of the turbulence intensity in the turbine wake, compared with the turbulence intensity of the incoming flow. As pointed out above, this can lead to increased fatigue loads in other wind turbines located downwind in a wind park. In the case of turbines located in free-stream flows, where the incoming flow 

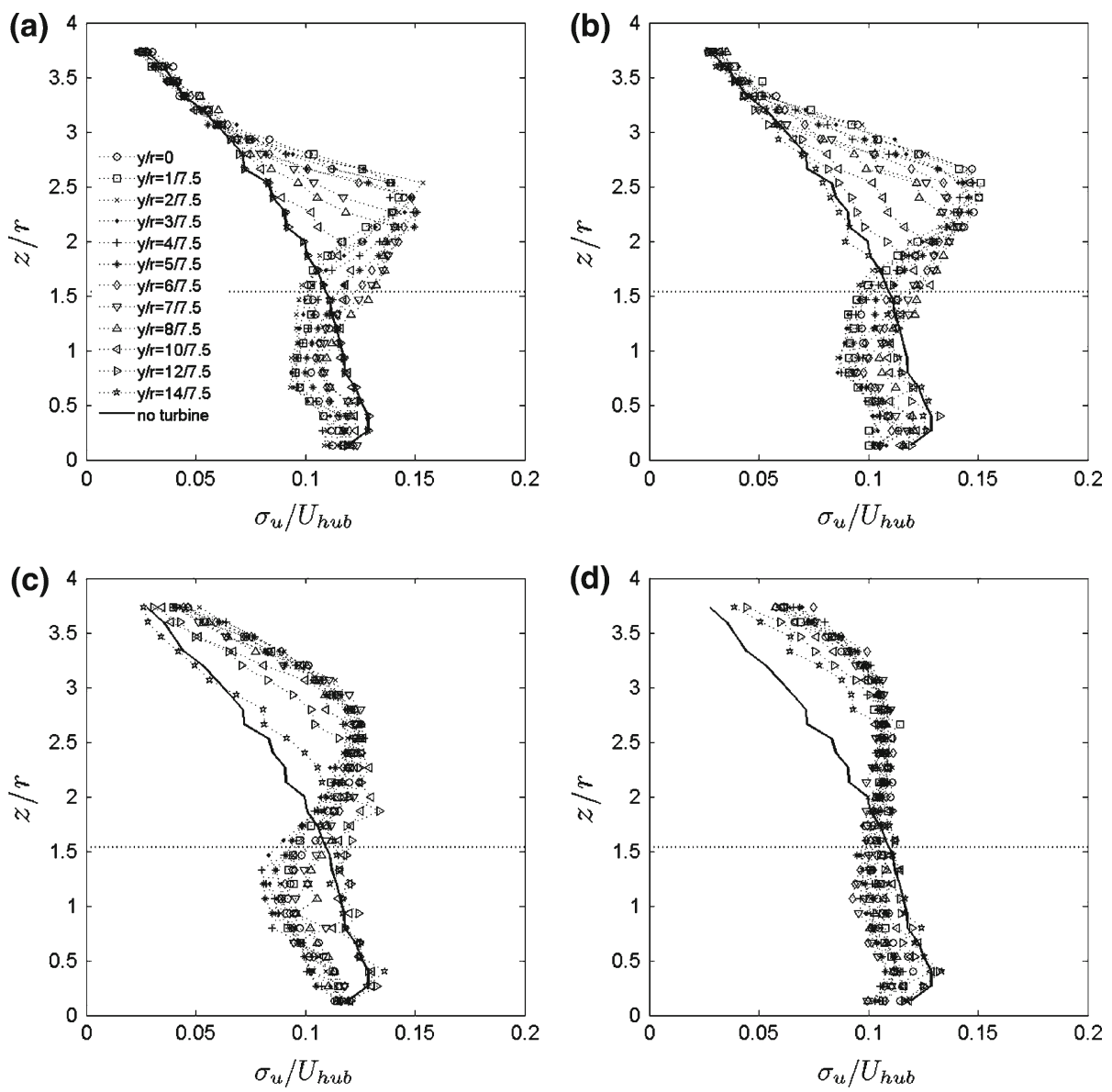

Fig. 4 Non-dimensional cross-sectional distribution of turbulence intensity at different downwind positions inside the wake over the rough surface. a $x / d=3 ; \mathbf{b} x / d=5 ; \mathbf{c} x / d=10$; and $\mathbf{d} x / d=15$. The horizontal line represents the turbine axis

is uniform, turbine wakes show an axisymmetric cross-sectional distribution of the turbulence intensity. In particular, one expects in the near wake $(x / d \leq 5)$ to find a peak in the turbulence intensity around the tip of the turbine wake, associated with the high turbulence levels produced by the helicoidal vortex that derive from turbine blade tip vortices (Hahm and Wubow 2006). Also in free-stream flow situations, both Ainslie (1988) and Frandsen (2003) associate the so-called far wake $(x / d \geq 5)$ with a Gaussian distribution of the cross-sectional profiles of turbulence intensity.

Having the wind turbine located in the boundary layer leads to a turbulence intensity distribution that is markedly non-axisymmetric and also non-Gaussian at all distances, as shown in Fig. 4. This behaviour is associated with the non-uniformity of the incoming flow and the presence of the surface. Near the upper edge of the wake, there is a strong enhancement of the turbulence intensity compared with the relatively low turbulence levels in the incoming flow. This is associated with the existence of a strong shear and the presence of helicoidal vortices at that location. However, the effect of the turbine leads to a reduction in the level of the turbulence intensity (compared to the relatively high turbulence levels near the ground) 

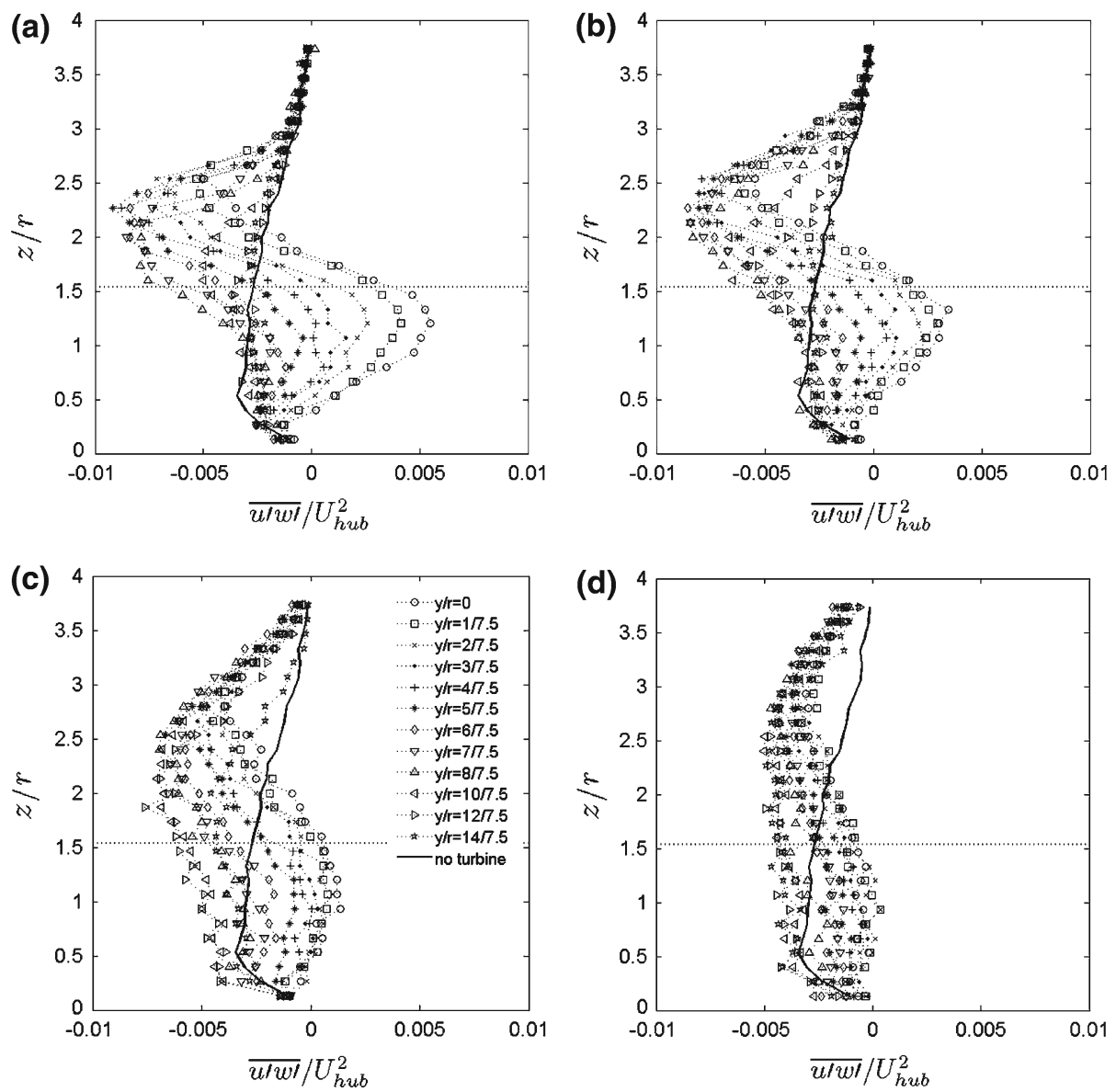

Fig. 5 Non-dimensional cross-sectional kinematic shear stress distribution over a rough boundary layer. a $x / d=3 ; \mathbf{b} x / d=5 ; \mathbf{c} x / d=10 ;$ and $\mathbf{d} x / d=15$. The horizontal lines represents the turbine axis

below the hub height. This can be explained by considering that in that region the mean shear (Fig. 3) and associated production of turbulence kinetic energy (not shown here) decrease with respect to the incoming flow.

Even though the turbulence enhancement effect decreases with distance, it remains evident even in the very far wake, at a distance $x / d=15$, a location at which turbulence intensity shows roughly a uniform distribution through the entire wake. Here, turbulent mixing has produced a uniform profile of turbulence intensity inside the wake.

A mean added turbulence intensity can be calculated at different downstream distances according to:

$$
\bar{I}_{+}=\frac{1}{A} \int_{A} I_{+}(x, y, z) d A
$$

where $A$ is the cross-sectional surface of the wake and $I_{+}$is calculated according to Eq. 6 . The distribution of the added turbulence intensity is shown in Fig. 8. Considering the non-axisymmetry of the turbulence intensity distribution shown in this figure, use of a 


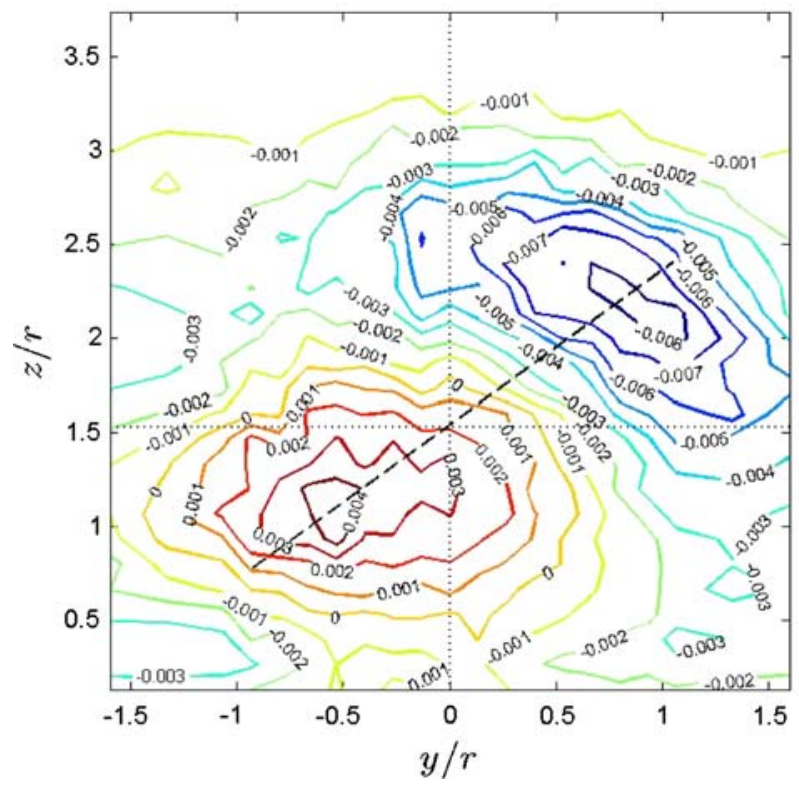

Fig. 6 Contour of non-dimensional kinematic shear stress distribution over a rough boundary layer at $x / d=5$

single value to represent the wake-averaged added turbulence intensity, $\bar{I}_{+}$, is not sufficient to account for the spatial variability of high turbulence intensity on downwind turbine loads. In a simple first attempt to include these non-axisymmetry effects, we propose to differentiate between two markedly different contributions to $\bar{I}_{+}$: a positive change (increase) $\bar{I}_{+}^{+}$in the upper part of the wake, and a negative change (decrease) $\bar{I}_{+}^{-}$in the lower part of the wake, of turbulence intensity relative to the background incoming-flow turbulence intensity. As in Eq. 14, these quantities are defined as:

$$
\begin{aligned}
& \bar{I}_{+}^{+}=\frac{1}{A_{+}} \int_{A_{+}} \sqrt{I_{\text {wake }}^{2}-I_{0}^{2}} d A, \text { for } I_{\text {wake }}>I_{0}, \\
& \bar{I}_{+}^{-}=\frac{1}{A_{-}} \int_{A_{-}} \sqrt{I_{0}^{2}-I_{\text {wake }}^{2}} d A, \text { for } I_{\text {wake }}<I_{0},
\end{aligned}
$$

where $A_{+}$and $A_{-}\left(=A-A_{+}\right)$are the cross-sectional areas where the change of turbulence intensity is positive $\left(I_{\text {wake }}>I_{0}\right)$ and negative $\left(I_{\text {wake }}<I_{0}\right)$, respectively. Also, indicators of the turbulent intensity are its maximum value and the value coincident with the hub level (often used as a reference).

Figure 9 shows the values of the average added turbulence intensity, $\bar{I}_{+}$, its positive $\left(\bar{I}_{+}^{+}\right)$ and negative $\left(\bar{I}_{+}^{-}\right)$contributions, and the maximum $\left(I_{+\max }\right)$ at different locations downwind of the turbine. The intensity is normalized with its maximum value at a distance of $x / d=5$. By examining the rate of change of these quantities with distance, it is inferred that in the far wake region, $5<x / d<15, \bar{I}_{+}^{+} \propto x^{-0.47}, \bar{I}_{+}^{-} \propto x^{-0.34}, \bar{I}_{+\max } \propto x^{-0.36}$, and $\bar{I}_{+} \propto x^{-0.46}$. In general these quantities decay as a power law of distance, with an exponent in the range $[-0.5,-0.3]$. This rate of decay is in good agreement with previously reported 

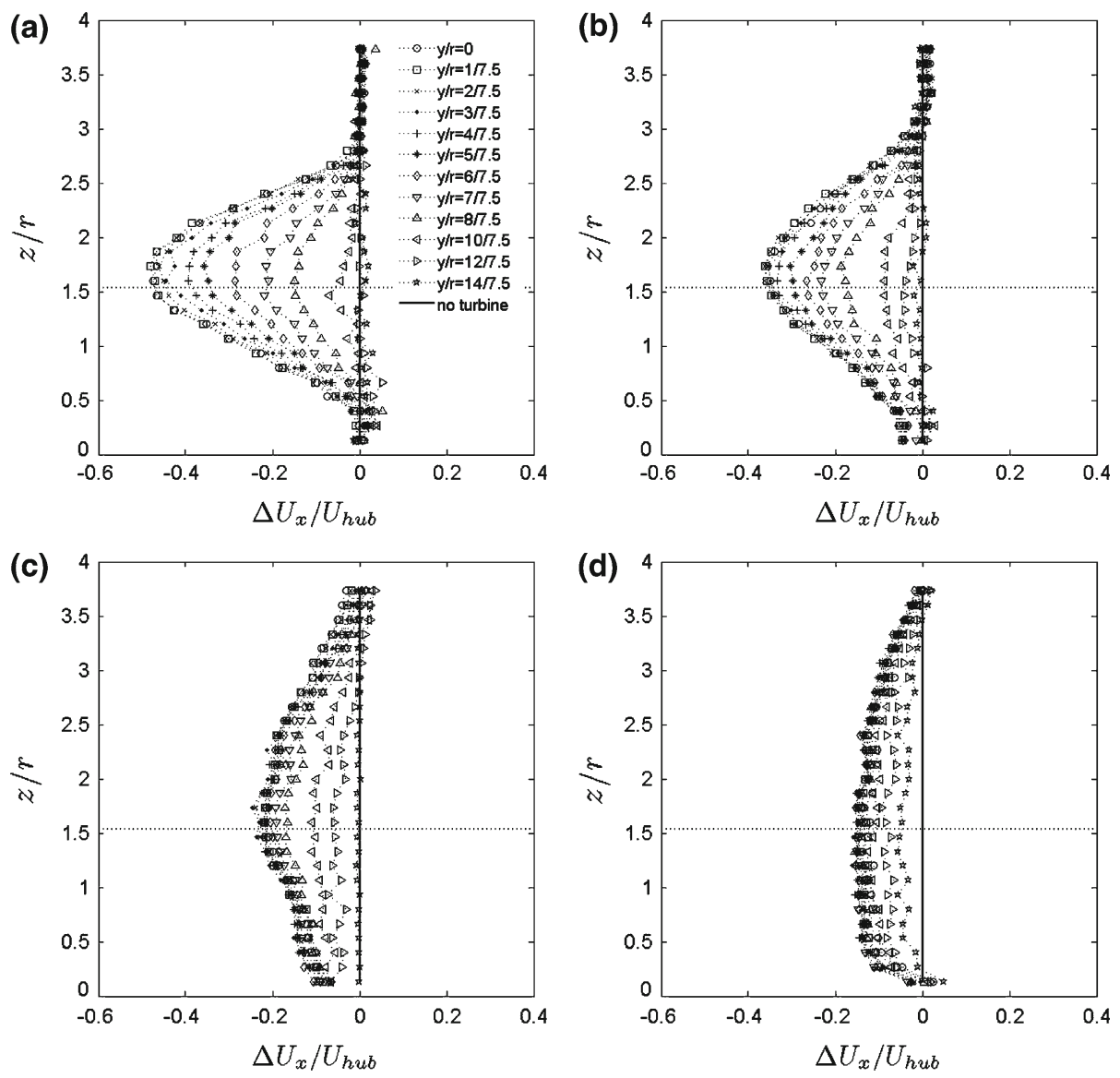

Fig. 7 Non-dimensional cross-sectional distribution of the velocity deficit at different downwind positions inside the wake over the rough surface. a $x / d=3 ; \mathbf{b} x / d=5 ; \mathbf{c} x / d=10$; and $\mathbf{d} x / d=15$. The horizontal line represents the turbine axis

results (e.g., Quarton and Ainslie 1989; Crespo and Hernandez 1996) for the case of the wake of a turbine located in a free-stream flow.

Figure 5 shows the spatial distribution of the kinematic shear stress $\left(\overline{u^{\prime} w^{\prime}}\right)$ for the different measurement locations. From this figure, it is clear that the turbine introduces kinematic stresses that are locally much larger in magnitude than the stresses in the incoming velocity field. In the near-wake region, at distances $x / d \leq 5$, there is a region above the turbine hub with large negative stress, and a lower region with large positive stress. As the wake grows with downwind distance, the relative change in the kinematic stress becomes smaller and affects a larger cross-sectional area. Notice that, as with the case of the velocity defect and the added turbulence intensity, the change in kinematic stress with respect to the incoming flow remains not negligible even in the very far wake, at $x / d \approx 15$. In order to illustrate the lack of symmetry of the turbulent stress with respect to the $y=0$ plane, Fig. 6 presents the distribution of the stress in an entire cross-sectional plane at $x / d=5$. This figure shows that, at that distance, the largest positive stress is located in the first quadrant (above the hub height and $y>0$ ), while the largest negative stress is found in the third quadrant (below the hub height and $y<0$ ). 

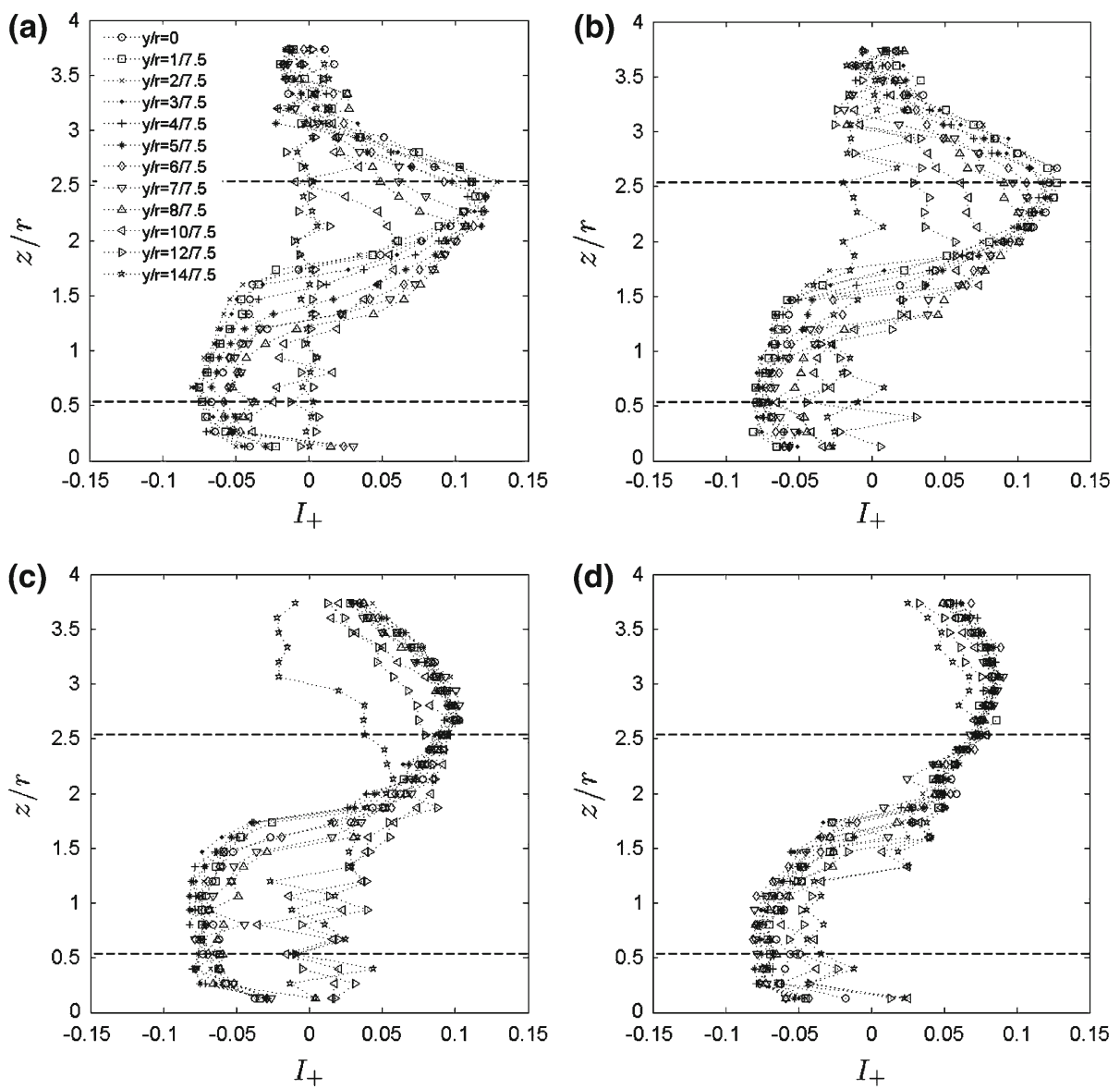

Fig. 8 Non-dimensional cross-sectional distribution of the added turbulence intensity at different downwind positions inside the wake over the rough surface. a $x / d=3 ; \mathbf{b} x / d=5 ; \mathbf{c} x / d=10$; and $\mathbf{d} x / d=15$. Horizontal lines represent the top tip and bottom tip levels

The distribution of streamwise velocity skewness, presented in Fig. 10, reveals that the effect of the turbine leads to a reduction in the magnitude of velocity skewness with respect to the incoming flow. In fact, skewness at different downwind locations inside the wake is slightly positive, which differs substantially from the negative skewness measured in the incoming flow away from the surface.

As pointed out by Carlin (2005), skewness plays a role in power generation. In fact, mean turbine power can be written as:

$$
\bar{P}=\frac{1}{2} \rho A \overline{U^{3}}=\frac{1}{2} \rho A \bar{U}^{3} K_{e}
$$

where

$$
K_{e}=\frac{1}{N \bar{U}^{3}} \sum_{i=1}^{N} U_{i}^{3}
$$




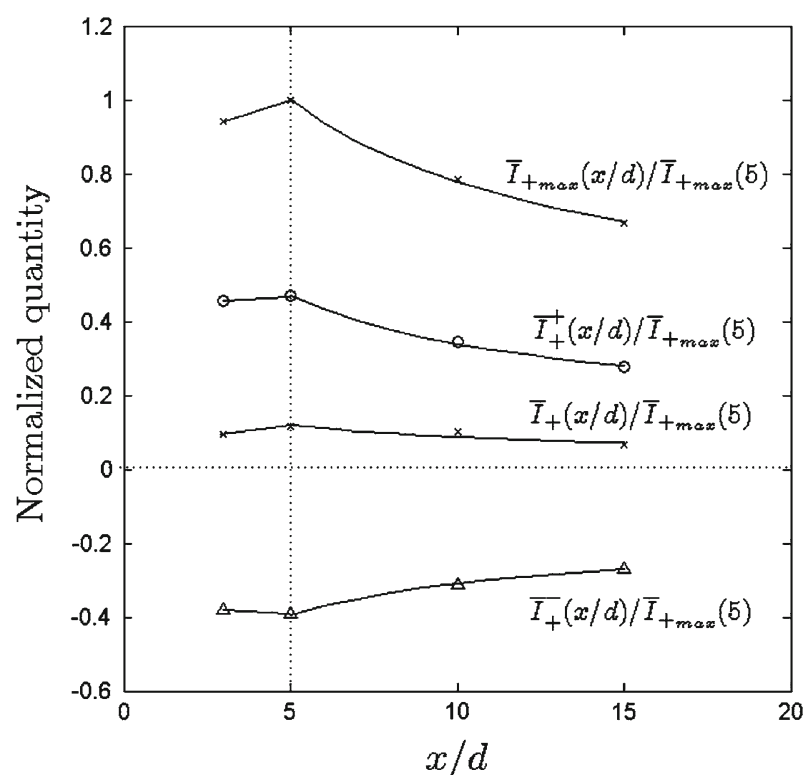

Fig. 9 Normalized added turbulence quantities as a function of distance. Rough case

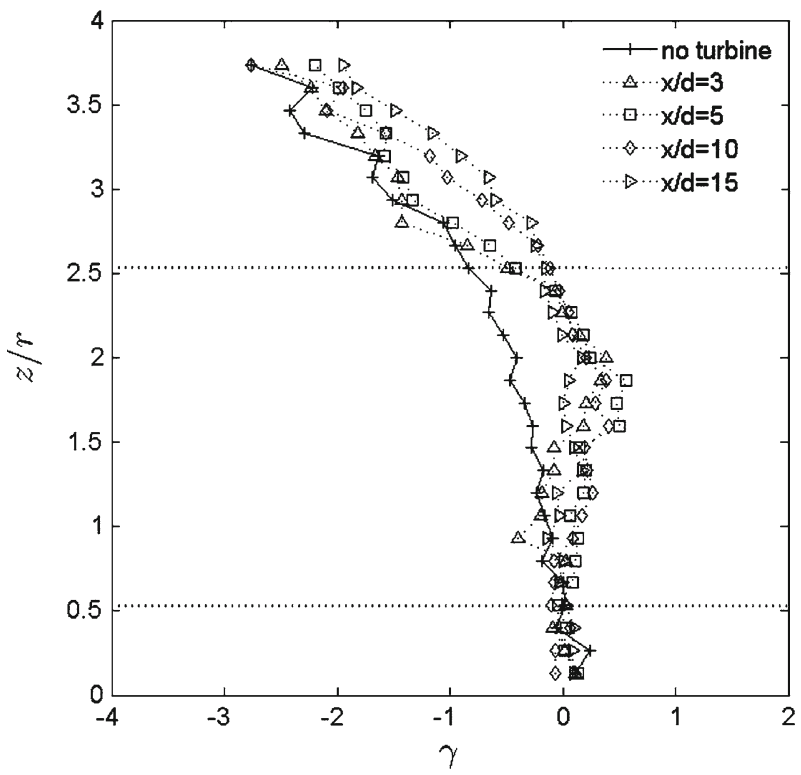

Fig. 10 Skewness of the streamwise velocity component at $y=0$ and different positions downwind $(x / d=3$, $5,10,15)$ and upwind of the turbine. Rough case. Horizontal lines represent the top tip and bottom tip levels 
for a discrete number of wind velocity data points. By expanding $\overline{U^{3}}$ using Reynolds decomposition,

$$
K_{e}=1+3 I_{u}^{2}+\gamma I_{u}^{3}
$$

where $\gamma$ is the skewness coefficient. According to Eq. 19, the skewness of the incoming wind can reduce or increase the mean power generation, depending on the sign of the skewness coefficient. It is important to point out that the turbine removed the negative skewness of the incoming velocity field away from the surface. As a result, velocity skewness in the wake will play a minor role (compared with velocity deficit and turbulence intensity) on the mean power from a turbine located in the wake of another turbine.

In order to better understand what range of scales contribute to the observed turbulence intensity changes induced by the wake, power spectra of the streamwise velocity are computed and shown in Fig. 11 for different positions in the wake. It includes, for comparison, the spectra of the incoming wind velocity (Fig. 11a). Comparison between spectra corresponding to the bottom-tip, hub-height and the top-tip levels at different downwind locations shows that the effect of the turbine on the turbulent intensities (enhancement at the top-tip level and reduction at the lower-tip level) are mostly associated with relatively large scales (frequencies lower than about $10 \mathrm{~Hz}$ ). The inertial subrange is found to grow with downwind distance and it is characterized by a similar level of energy at any position inside the turbine wake. Note that very close to the wind turbine, at a distance $x / d=1$, the velocity spectrum calculated at the top-tip level has a clear peak associated with the presence of tip vortices (Fig. 11b). The peak occurs at a frequency of $\approx 60 \mathrm{~Hz}$, which coincides with three times the frequency of rotation of the rotor, or the frequency of consecutive blades.

The peak is not observed at the bottom-tip level, where the turbulence levels of the incoming flow are relatively high. In the wake, at $x / d=15$, the energy content is very similar at all scales and still substantially different from that in the incoming flow. This is consistent with the nearly uniform profile of turbulence intensity measured at that location and shown in Fig. 4d.

\subsection{Wake in a Smooth-Wall Boundary Layer}

In this section, turbulence measurements collected in the wake of a wind turbine located in a boundary layer over a smooth surface are presented. In particular, Figs. 12 and 13 show the cross-sectional distributions of mean streamwise velocity deficit $\left(\Delta U_{x}=U(x>\right.$ $0, y, z)-U(x<0, y, z))$ and the added turbulence intensity $\left(I_{u}\right)$ measured with the cross-wire anemometer at different locations downwind of the turbine $(x / d=3,5,10$ and $15)$, similar to the rough case.

Comparison of these figures with their counterparts for the rough case reveals an important effect of surface roughness on the degree of non-axisymmetry of the turbine wake and its evolution. The wake over the smooth surface shows a relatively smaller departure from axisymmetry compared with the rough surface case even when it has reached the surface. This can be explained considering that the incoming velocity field is more uniform compared with the rough case (see Fig. 2). Since wakes are superimposed on the incoming velocity distribution, the more uniform the incoming flow at the turbine level, the smaller the departure from axisymmetry of the wake. In the limiting case of a uniform incoming flow (e.g., a turbine placed in a free-stream flow), the turbine wake is axisymmetric (Vermeer et al. 2003; Medici and Alfredsson 2006). 

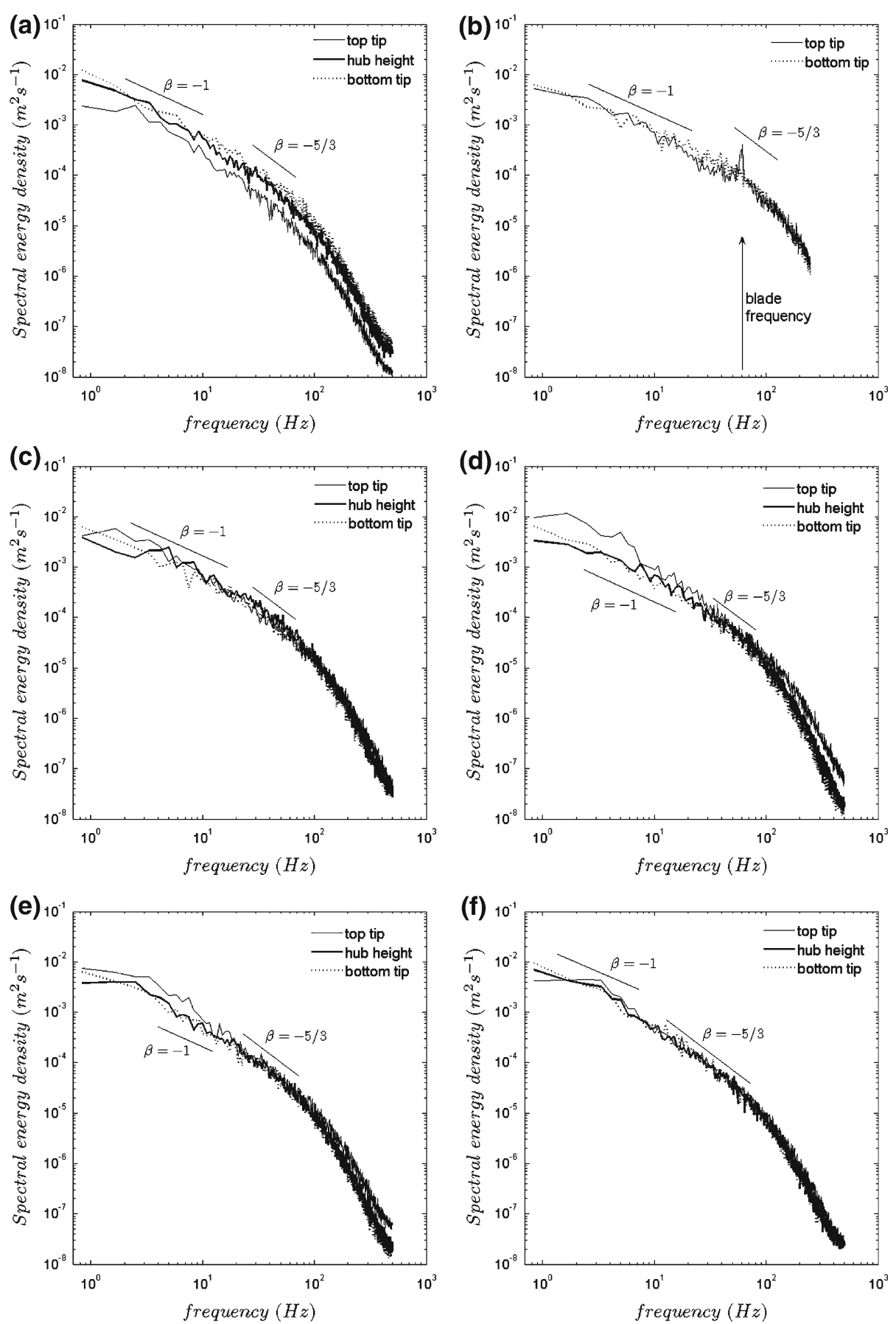

Fig. 11 Power spectrum of the streamwise velocity at different heights (top-tip level, hub height and bottom-tip level) and different downwind positions in the center plane of the wake $(y=0)$. a Without turbine, b $x / d=1, \mathbf{c} x / d=3, \mathbf{d} x / d=5$, e $x / d=10$ and $\mathbf{f} x / d=15$ 

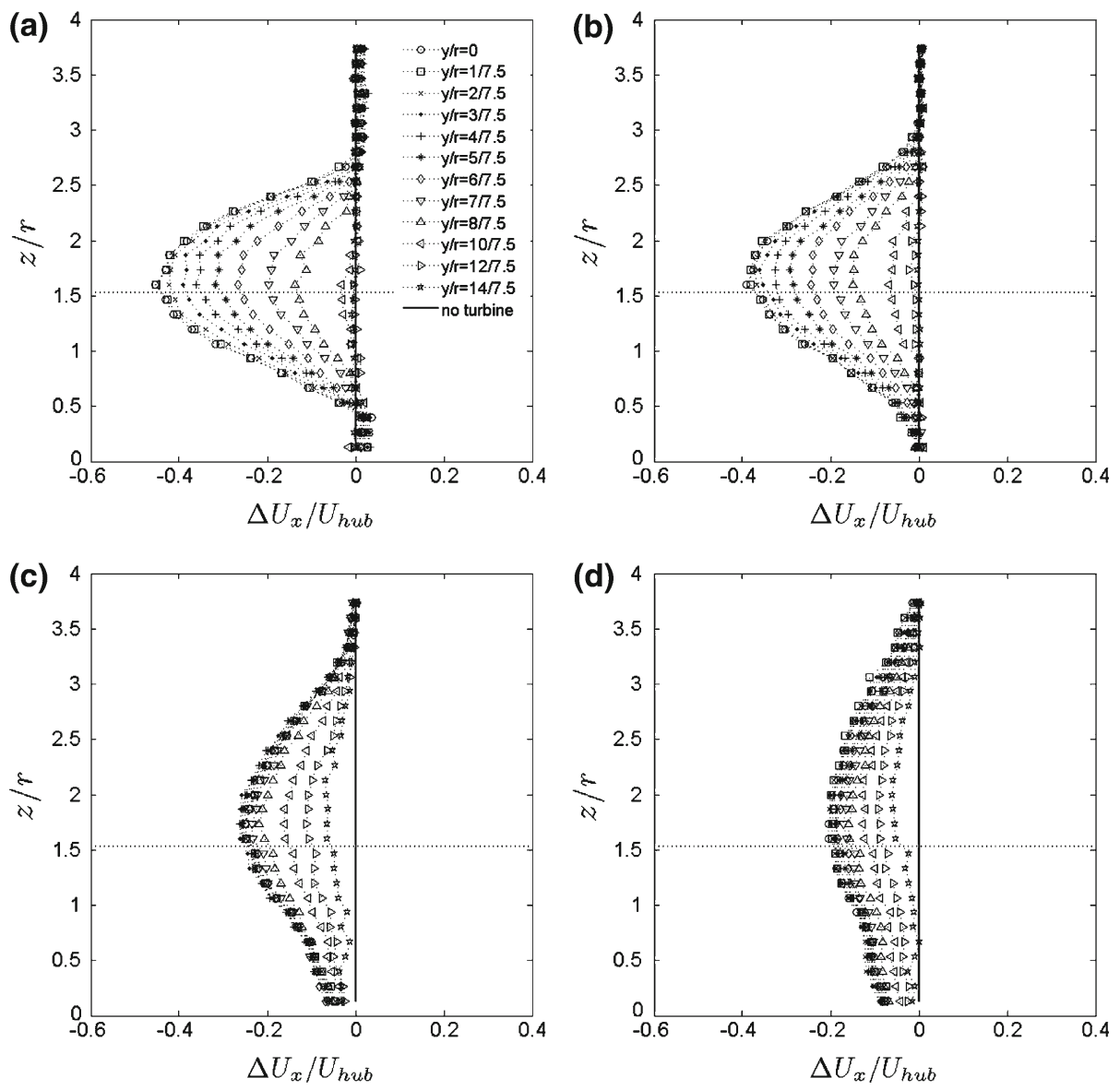

Fig. 12 Non-dimensional cross-sectional distribution of the velocity deficit at different downwind positions inside the wake over the smooth surface. $\mathbf{a} x / d=3 ; \mathbf{b} x / d=5 ; \mathbf{c} x / d=10$; and $\mathbf{d} x / d=15$. The horizontal lines represents the turbine axis

The velocity deficit $\left(\Delta U_{x}\right)$, shown in Fig. 12, reveals that the turbine wake touches the surface at a distance $x / d$ within the range $[5,10]$, which is longer than the rough case, where this distance is $x / d<5$ (Fig. 7). This observation shows clearly that the cross-sectional expansion of the wake is affected by the surface roughness. From Fig. 12, it is possible to notice that the height of the axis of symmetry increases with distance. Overall, in the smooth surface case, the magnitude of the velocity deficit in the wake is larger than in the rough surface case. Also from Fig. 12, it is noted that this velocity deficit decreases with downwind distance at a slower rate compared to the rough case.

Added turbulence intensity profiles in the turbine wake show important differences with respect to the rough case (Figs. 13 and 8, for the smooth and rough cases, respectively). Even though in both cases the non-uniformity of the incoming flow leads to a non-axisymmetric distribution of the added turbulence intensity in the turbine wake, this non-axisymmetry is smaller over the smooth surface. This is obvious when considering the relative importance of the negative change of turbulence intensity, $\bar{I}_{+}^{-}$, as defined by Eq. 16 . In particular, the reduction of turbulence intensity in the lower part of the wake with respect to the incoming 

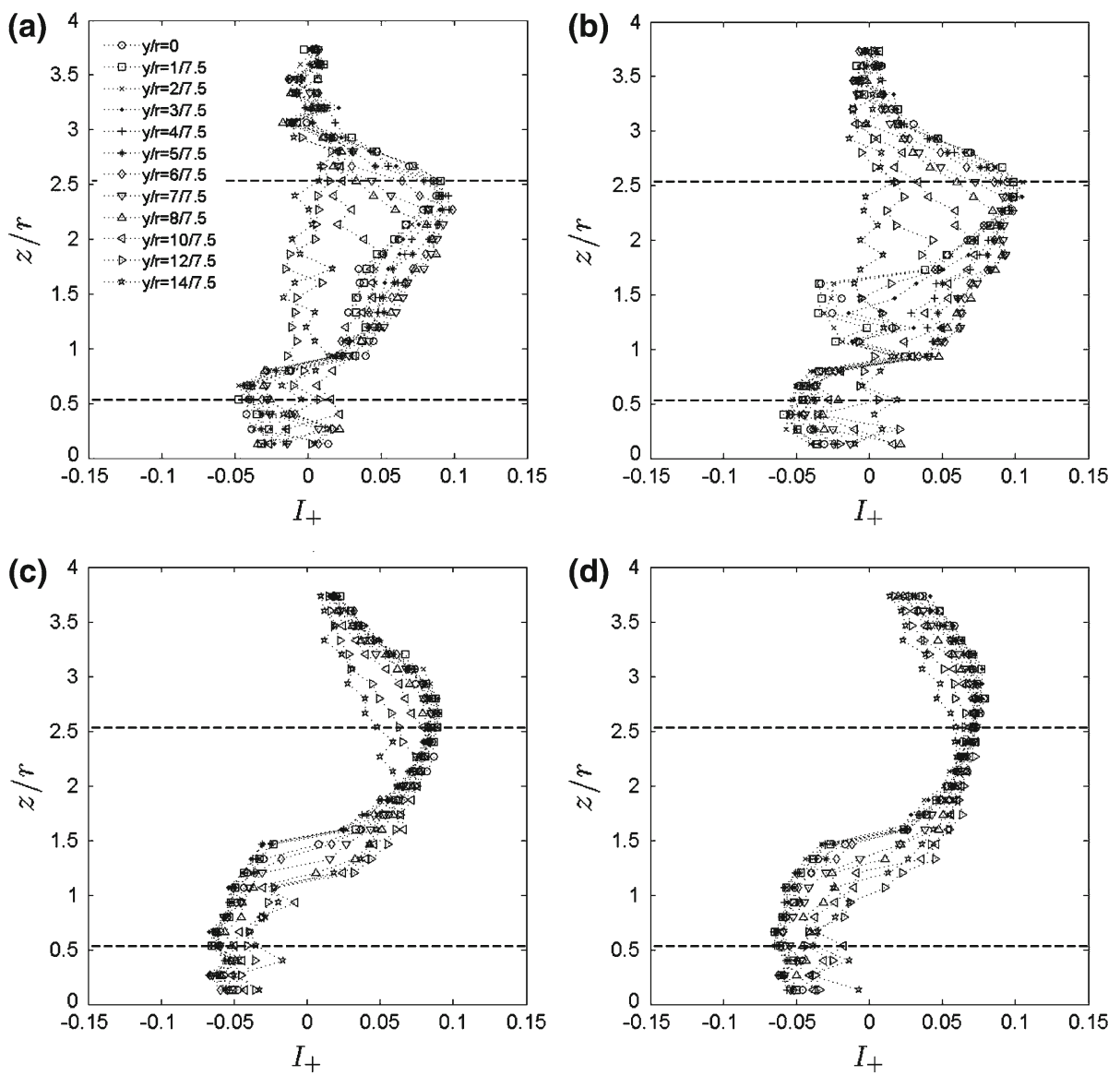

Fig. 13 Non-dimensional cross-sectional distribution of the added turbulence intensity at different downwind positions inside the wake over the smooth surface. a $x / d=3$; b $x / d=5 ; \mathbf{c} x / d=10$; and d $x / d=15$. Horizontal lines represent the top tip and bottom tip levels

flow is relatively smaller over the smooth surface. In contrast to the rough case, at distances $x / d \approx 5, \bar{I}_{+}^{-}$is confined to lower heights $(z / r \leq 1)$ compared with the rough case for which $\bar{I}_{+}^{-}$extends up to a height of $z / r \approx 1.75$. Over the smooth surface, $\bar{I}_{+}^{-}$grows up to $z / r \approx 1.5$ at larger distances.

It was found (not shown here) that the average added turbulence intensity, $\bar{I}_{+}$, its positive $\left(\bar{I}_{+}^{+}\right)$and negative $\left(\bar{I}_{+}^{-}\right)$contributions, and its maximum $\left(I_{+\max }\right)$ change with downwind distance as $\bar{I}_{+} \propto x^{-0.7}, \bar{I}_{+}^{+} \propto x^{-0.41}, \bar{I}_{+}^{-} \propto x^{-0.12}$ and $\bar{I}_{+\max } \propto x^{-0.22}$.

The qualitative distribution of the kinematic shear stress $\overline{u^{\prime} w^{\prime}}$ (result not shown here) is comparable with that over the rough surface (Fig. 5). As in that case, the cross-sectional distribution of the stress is also antisymmetric, with the axis of symmetry rotated approximately $45^{\circ}$. However, it is important to remark that the magnitude of the kinematic stress is smaller than the one measured in the rough case, which highlights the importance of considering the incoming flow so as to understand the distribution of kinematic stresses and other turbulence quantities in turbine wakes. 


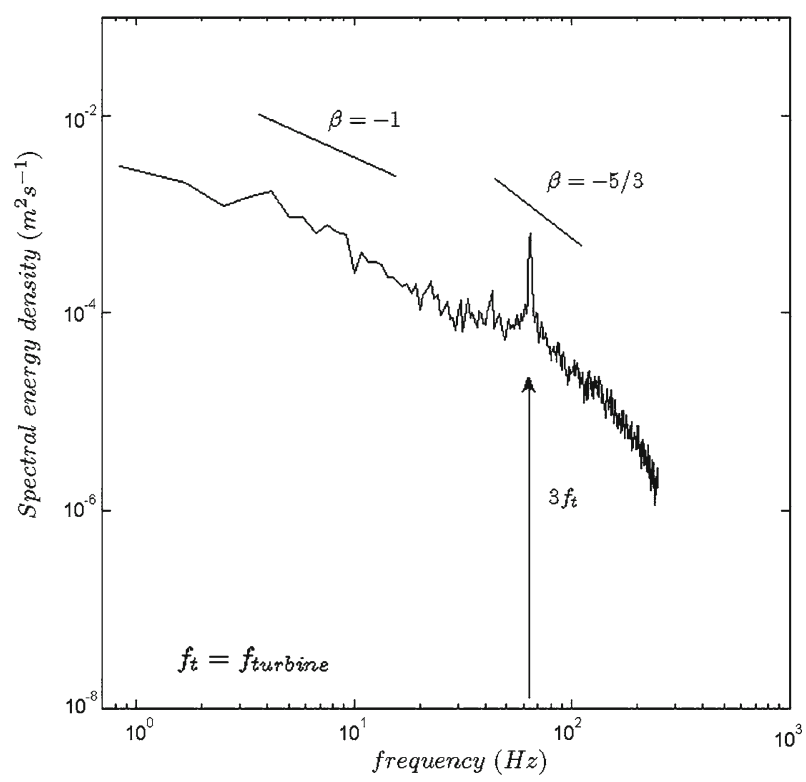

Fig. 14 Power spectrum of streamwise velocity at the top tip and $x / d=1$

Skewness and spectra of the streamwise wind velocity component calculated in the wake of the turbine over the smooth surface (not shown here) have the same qualitative behaviour as discussed in Sect. 3.1 for the rough case. Nevertheless, the signature of the tip vortices near the turbine $(x / d=1)$ and at the top-tip level is stronger in the smooth case due to the relatively lower background turbulence level in the incoming flow with respect to the rough case (Fig. 14).

\section{Summary}

A wind-tunnel experiment was performed to study the effects of boundary-layer turbulence and surface roughness on the structure of the wake developed downwind of a model turbine. Hot-wire anemometry was used to obtain detailed measurements of wind velocity, turbulence intensity and kinematic stress over several cross-sections at different positions downwind of the turbine. Skewness coefficients and power spectra of the streamwise velocity were also calculated to obtain a better understanding of the turbulence inside the wake. The turbine was placed in a boundary layer developed over both rough and smooth surfaces under neutral conditions. Special emphasis was placed on quantifying the magnitude and spatial distribution of the velocity deficit and the enhancement of the turbulence intensity in the turbine wake with respect to the incoming boundary-layer flow. These quantities are important factors affecting turbine power output and fatigue loads in wind parks.

In general, the vertical inhomogeneity of the incoming boundary-layer flow, which is modulated by the surface roughness, plays an important role on the distribution of turbulence properties within the turbine wake. In contrast to the axisymmetric structure of wakes developed in free-stream (uniform) flows, the incoming flow inhomogeneity leads to a break down of axisymmetry of the turbulence statistics. It is found that the departure from axisymmetry increases with surface roughness due to the increased non-uniform distribution of velocity and turbulence levels at turbine level induced by the roughness. 
As expected, the velocity deficit and the turbulence enhancement decrease with distance as the wake expands. However, these effects remain not negligible even in the very far wake, at a distance of fifteen turbine diameters downwind of the turbine location. Even though the velocity distribution is not axisymmetric, the velocity deficit $\left(\Delta U_{x}=U(x>\right.$ $0, y, z)-U(x<0, y, z))$ is nearly axisymmetric except near the surface downwind of the location where the wake touches the surface. The distance between the turbine and that location is found to decrease with increasing surface roughness. The quasi-axisymmetric distribution of the velocity deficit has important implications for the development of models for the velocity distribution and, in particular, the possible extension of existing models for velocity deficit of wakes in free-stream flows.

The wind turbine induces a large enhancement of turbulence levels (positive change in the turbulence intensity) in the upper part of the wake. In the near-wake region, this turbulence enhancement is more pronounced near the turbine edge, which can be explained by the strong shear and the presence of helicoidal vortices (derived from tip vortices) in that region. These vortices induce turbulence levels that are relatively large compared with the background boundary-layer turbulence levels at that height, thus leading to large positive change in turbulence intensity. It was also found that turbulence intensity decreases (loss of turbulent kinetic energy with respect to the incoming flow) in the lower region of the wake due to the reduction of mean shear and production of turbulence kinetic energy with respect to the incoming flow. This effect is more notorious in the rough surface case.

In the far wake region $(x / d \geq 5)$, the added turbulence intensity, its positive and negative contributions, as well as the local maximum, decrease with downwind distance following a power law with exponents in the range of $[-0.5,-0.3]$ for the rough surface case. These values are in good agreement with previously reported results for the case of turbines in free-stream flows (Quarton and Ainslie 1989; Crespo and Hernandez 1996). The exponents of decay in the smooth surface case showed more variability. The non-axisymmetric distribution of the turbulent intensity is expected to have an important role in the fatigue loads acting on a turbine placed in the wake of other turbines in wind parks.

Power spectral density of the streamwise velocity was calculated in order to better understand the distribution of the added turbulence levels in the wake as a function of scale. The results indicate that in the far wake the added turbulence intensity is associated with relatively large turbulence scales in the flow. As with the turbulence intensity distribution, the increase in spectral density is also found to be largest near the upper edge of the wake. Consistent with the turbulence intensity distribution, these differences in the energy content are maximum at $x / d \approx 5$, which corresponds to the transition zone between the near wake and far wake regions. Also consistent with the relatively 'uniform' added turbulence intensity distribution in the very far wake region $(x / d \approx 15)$, velocity spectra show little differences for different positions within the wake in that region. The effect of the tip vortices on the turbulent kinetic energy of the flow is clearly observed at the top-tip level at $x / d=1$. There, the power spectral density reveals a jump in the energy at a frequency coincident with the blade rotation. Due to the higher energy levels in the incoming flow near the surface, that peak is not clear at the bottom-tip level.

Future work will consider the effect of atmospheric stability on the turbulent structure and behaviour of the wake. Also, other factors affecting the characteristics of the incoming flow will be considered. These include surface inhomogeneity (e.g., roughness and temperature transitions), topography and the presence of multiple turbines in a wind farm configuration.

Acknowledgements The authors gratefully acknowledge the assistance of Toni Calderer and James Tucker during the course of the experiments. Funding was provided by NSF (grant EAR-0537856), NASA (grant 
NNG06GE256) and customers of Xcel Energy through a grant (RD3-42) from the Renewable Development Fund. Computing resources were provided by the University of Minnesota Supercomputing Institute.

Open Access This article is distributed under the terms of the Creative Commons Attribution Noncommercial License which permits any noncommercial use, distribution, and reproduction in any medium, provided the original author(s) and source are credited.

\section{References}

Ainslie J (1988) Calculating the flowfield in the wake of wind turbines. J Wind Eng Ind Aerodyn 27:213-224

Binh L, Ishihara T, Van Phuc P, Fujino Y (2008) A peak factor for non-Gaussian response analysis of wind turbine tower. J Wind Eng Ind Aerodyn 96:2217-2227

Bruun HH (1995) Hotwire anemometry, principles and signal analysis. Oxford Univ Press, UK, 507 pp

Burton T, Sharpe D, Jenkins N, Bossanyi E (1995) Wind energy handbook, 1st edn. Wiley, Chichester, 617 pp

Carlin P (2005) A suggested modification to a common expression for the energy content of a wind data sample. Wind Energy 8:477-480

Carper M, Porté-Agel F (2008) Subfilter-scale fluxes over a surface roughness transition, Part I: measured fluxes and energy transfer rates. Boundary-Layer Meteorol 126:157-179

Crespo A, Hernandez J (1996) Turbulence characteristics in wind turbine wakes. J Wind Eng Ind Aerodyn 61:71-85

Crespo A, Hernandez J, Frandsen S (1999) Survey of modelling methods for wind turbine wakes and wind farms. Wind Energy 2:1-24

Frandsen S (2003) Turbulence and turbulence-generated fatigue loading in wind turbine clusters. Wind Energy Department, Ris $\varnothing$ National Laboratory, Roskilde, Denmark

Frandsen S, Thogersen M (1999) Integrated fatigue loading for wind turbines in wind farms by combining ambient turbulence and wakes. J Wind Eng 23:327-339

Hahm T, Wubow S (2006) Turbulent wakes in wind farm configuration. Paper presented at the European Wind Energy conference \& exhibition, Athens, Greece, 27 February-2 March 2006

Hassan U (1992) A wind tunnel investigation of the wake structure within small wind turbine farms. E/5A/CON/5113/1890. UK Department of Energy, ETSU

Medici D, Alfredsson P (2006) Measurement on a wind turbine wake: 3D effects and bluff body vortex shedding. Wind Energy 9:219-236

Ohya Y (2001) Wind-tunnel study of atmospheric stable boundary layers over a rough surface. BoundaryLayer Meteorol 98:57-82

Quarton D, Ainslie J (1989) Turbulence in wind turbine wakes. J Wind Eng 14:15-23

Rosen A, Sheinman Y (1996) The power fluctuations of a wind turbine. J Wind Eng Ind Aerodyn 59:51-68

Sheinman Y, Rosen A (1992) A dynamic model of the influence of turbulence on the power output of a wind turbine. J Wind Eng Ind Aerodyn 39:329-341

Vermeer L, Sorensen J, Crespo A (2003) Wind turbine wake aerodynamics. Prog Aerospace Sci 39:467-510

Vermeulen P (1980) An experimental analysis of wind turbine wakes. In: Third international symposium on wind energy systems, BHRA 